\title{
Comparative Study on Electronic, Emission, Spontaneous Property of Porous Silicon in Different Solvents
}

\author{
M. Naziruddin Khan, ${ }^{1}$ M. A. Majeed Khan, ${ }^{1}$ A. S. Al Dwayyan, ${ }^{2}$ and J. Puzon Labis ${ }^{1}$ \\ ${ }^{1}$ King Abdullah Institute for Nanotechnology, King Saud University, Riyadh 11451, Saudi Arabia \\ ${ }^{2}$ Department of Physics \& Astronomy, College of Science, Riyadh 11451, Saudi Arabia
}

Correspondence should be addressed to M. Naziruddin Khan; mnkhan_phy@yahoo.com

Received 10 April 2014; Accepted 25 May 2014; Published 15 October 2014

Academic Editor: William W. Yu

Copyright (c) 2014 M. Naziruddin Khan et al. This is an open access article distributed under the Creative Commons Attribution License, which permits unrestricted use, distribution, and reproduction in any medium, provided the original work is properly cited.

\begin{abstract}
Luminescent porous silicon (Psi) fabricated by simple chemical etching technique in different organic solvents was studied. By quantifying the silicon wafer piece, optical properties of the Psi in solutions were investigated. Observation shows that no photoluminescence light of Psi in all solvents is emitted. Morphology of Psi in different solvents indicates that the structure and distribution of Psi are differently observed. Particles are uniformly dispersive with the sizes around more or less 5-8 nm. The crystallographic plane and high crystalline nature of Psi is observed by selected area diffraction (SED) and XRD. Electronic properties of Psi in solutions are influenced due to the variation of quantity of wafer and nature of solvent. Influence in band gaps of Psi calculated by Tauc's method is obtained due to change of absorption edge of Psi in solvents. PL intensities are observed to be depending on quantity of silicon wafer, etched cross-section area on wafer surface. Effects on emission peaks and bands of Psi under temperature annealing are observed. The spontaneous signals of Psi measured under high power Pico second laser $355 \mathrm{~nm}$ source are significant, influenced by the nature of solvent, pumped energy, and quantity of Si wafer piece used in etching process.
\end{abstract}

\section{Introduction}

Silicon based nanocrystallines/nanoporous are some new photoelectronic and informational materials developed rapidly in recent years. For a long time, silicon has been considered unsuitable for optoelectronic applications because bulk silicon emits hardly any useful light due to its indirect band gap nature [1]. This opinion was deeply changed after the discovery of bright emission from porous silicon and nanocrystals. The possibility of constructing optoelectronic devices, Si based integrated circuits [2], has generated tremendous interest in the preparation and characterization of light emitting silicon nanoparticles since its photoluminescence was observed first time by Canham in 1990 [3]. Since then many workers began significant interest in light emitting porous silicon [4].

Due to the fact that the luminescence properties of $\mathrm{Si}$ nanoparticles are size dependent, multicolors which have exciting potential applications as fluorescent tags for biological imaging [5-7] and a great variety of nanoparticles are currently used for bioanalysis including quantum dots [8]. Silicon nanoparticles are much brighter and more stable to photobleaching [9] than the organic dyes [10] with broader excitation spectra, so that emission at multiple wavelengths (from particles of different sizes) can be excited by a single source. On the other hand, the nontoxic behavior of silicon nanoparticles is important for the application in pollution [11]. Moreover, the use of nanoporous silicon/nanocrystals as an active medium for light emitting devices (LEDs or injection laser) also remains an open issue for basic research, even if for various applications in waveguides, photodetectors, solar cells, gas sensors [12-14], and so forth. One of the most challenging goals is to achieve optical gain and/or lasing in silicon for optoelectronic circuits because the key device is the light source-laser. In this direction, workers approached toward the silicon nanocrystals (nc-Si) embedded in an insulator matrix, usually $\mathrm{SiO}_{2}$ matrix [15], because of their photoemission properties and it is believed that the interface between the nc-Si and the oxide matrix plays a crucial role of controlling the optoelectronic properties. One of the 
promising techniques to form such a nc-Si structure is the implementation of silicon ions into a $\mathrm{SiO}_{2}$ matrix followed by thermal annealing $[16,17]$. But silicon based nanocrystals with strong light emission prepared at low temperature in a host matrix with the smallest band gap are tremendously interested. Recently the direct encapsulation of nc-Si/Psiderived nanoparticles in sol gel was successfully employed in order to maintain the PL intensity reported $[18,19]$. Because, demonstration of Psi/nc-Si in sol gel is easily manageable, that enables the fabrication of samples with high densities of silicon nanoparticles for optoelectronics purposes. Due to being greatly important to optical stability of Psi/nc-Si within sol gel matrix in view of light stimulation, recently systematic studies were done by our group especially on $\mathrm{PL}$ and structural properties of the NCs powder $[20,21]$, nanoporous silicon $[22,23]$ in sol gel host. Although porous silicon could stabilize in the sol gel active medium, weak emission and spontaneous signals were obtained due to low concentration of Psi colloidal solutions. It is required to obtain high densities of Psi colloidal solutions for the amplification light. From the point of above, by quantifying the silicon wafer piece in etching process, efforts were made to assemble the high densities of Psi in solvents and understand the effects on optical properties especially on PL due to quantity of silicon wafer piece.

\section{Experimental Method}

Several methods have been used to obtain Psi with different pore sizes ranging from micro- to nanometers. Electrochemical etching is widely used to produce Si crystals by applying an electrical current to an electrolyte solution containing hydrofluoric acid (HF) $[24,25]$. On the other hand, the efficient room temperature luminescence in Psi produced by chemically etching method (without applying current) was first reported $[26,27]$. In present work, nanoporous silicon were fabricated by using a simple chemical etching process in a highly catalyst solution of $40 \% \mathrm{HF}$, hexachloroplatinic (IV) acid $\left(\mathrm{H}_{2} \mathrm{PtCl}_{6}\right)$, deionised water and etchant solution of methanol, $\mathrm{HF}$, and $30 \% \mathrm{H}_{2} \mathrm{O}_{2}$ to produce crystalline $\mathrm{Si}$ into ultrasmall nanoparticles. In fact, $\mathrm{HF}$ is highly reactive with silicon oxide and $\mathrm{H}_{2} \mathrm{O}_{2}$ catalyses the etching that able to produce smaller particles from the surface of silicon wafer. Moreover, the oxidative nature of the peroxides produces high chemical and electronic quality samples. For fabrication, the catalyst solution was prepared by taking the $13.5 \mathrm{~mL}$ DI water, $5 \mathrm{~mL} \mathrm{HF}$, and $1.5 \mathrm{~mL}$ of chloroplatinic solution. The etchant solution was prepared using the $10 \mathrm{~mL}$ methanol, $5 \mathrm{~mL} \mathrm{HF}$, and $10 \mathrm{~mL}$ of $\mathrm{H}_{2} \mathrm{O}_{2}$. The catalyst and etchant solution were filled in two small polypropylene tanks. Single $\mathrm{P}$ type boron doped Si wafers $(100,1-10 \mathrm{ohm})$ were cut into small rectangular pieces $(1 \times 4 \mathrm{~cm})$ in area which was ultrasonically cleaned with acetone before use, dried with $\mathrm{N}_{2}$ gas, and put in a polypropylene tank filled catalyst solution about $15 \mathrm{~min}$. The samples were then rinsed with deionized water and dried in blowing $\mathrm{N}_{2}$ gas. The subsequent chemical etching was made for 15 second of each sample in the etchant solution. Finally the etched wafers were rinsed in isopropanol each time after the etching and dried slowly with $\mathrm{N}_{2}$ gas. The pulverized wafer pieces were transferred into a small disk filed with different solvents of the same volume such as THF, 1,4 Dioxane, DMSO, DMF, $\mathrm{CCl}_{4}$, cyclohexane, chloroform, methanol, and ethanol. Then the wafer pieces in solvents were treated sonication in an ultrasound bath to disperse the $\mathrm{Si}$ nanoparticles. Samples were centrifuged about 5-10 minutes and exposed to an UV lamp (Cole-Parmer, USA) at $365 \mathrm{~nm}$ to detect the light emitting. X-ray diffraction (XRD) patterns of the dried Psi were obtained at room temperature using a PANalytical X'Pert X-ray diffractometer. The morphology of the samples was inspected by field emission scanning electron microscope (FESEM, JEOL, JSM-6380LA). Samples for transmission electron microscopy (TEM) were prepared by depositing a drop of colloidal Psi in solvents onto a carbon-coated copper grid. Images were obtained on a high resolution TEM (JEOL, JEM2100F). Electronic and emission spectra were measured in UV-Visible-NIR (Jasco 670 spectrophotometer) and Lumina luminescence Spectrometer (Thermo). The spontaneous spectra were obtained in High Power Pico Second Tunable Laser System (Lotti III) with ICCD Spectrograph (Andor).

\section{Results and Discussion}

To understand the effect on the various properties of porous silicon due to densities or concentration, an equal size of $1 \times 4 \mathrm{~cm}$ wafer pieces were cut and used in etching process. The different quantity of etched wafer pieces was employed in an equal volume $(15 \mathrm{~mL})$ of each solvent. Among the colloidal porous silicon solutions, Psi in THF, dioxane, DMSO, $\mathrm{CCl}_{4}$, cyclohexane, and chloroform exhibited the luminescent under the exposure of UV lamp. But Psi in $\mathrm{CCl}_{4}$, cyclohexane, and chloroform solution was found to be unstable and decayed the luminescent light within few days later. Digital image of luminescent Psi solutions under the excitation of UV lamp is shown in Figure 1.

In present study, only those luminescent $\mathrm{Si}$ solutions such as Psi in THF, dioxane, and DMSO were systematically carried out using different optical techniques. X-ray diffraction (XRD) pattern of porous silicon particles on the etched silicon wafer piece was inspected to confirm the purity and crystallographic structure. The XRD pattern of the Psi on the surface of etched silicon wafer is shown in Figure 2. The pattern reveals that major peaks of silicon correspond to the crystallographic plane (111), (220), (311), (400), (331), (422), and (511) as identified using the standard data JCPDS (Card No.027-1402). The prominent peak at $28.9^{\circ}$ is assigned to the (111) plane of $\mathrm{Si}$ as identified using the standard data. Due to the reduction in crystal size to nanometric scale, a broadening of diffraction peaks is observed and the width of the peak which is also directly correlated to the size of the nanocrystalline domains [28]. The size of the nanocrystallite is found to be $\sim 10.68 \mathrm{~nm}$. The luminescent and nonluminescent Psi solution is listed in Table 1 . The common physical properties data of solvents was extracted from [29]. 
TABLE 1: List of physical properties of organic solvents with PL.

\begin{tabular}{lcccccccc}
\hline Solvents & $\mathrm{BP}^{\circ} \mathrm{C}$ & Dipole moment & Dielectric constant & Polarity & Porous silicon (Psi) & PL & Stability & Solvent category \\
\hline Ethanol & 78 & 1.69 & 24.5 & 5.2 & Psi & No & Aggregate & Polar protic \\
Methanol & 65 & 1.70 & 32.7 & 5.1 & Psi & No & Aggregate & Polar protic \\
THF & 66 & 1.75 & 7.58 & 4 & Psi & Yes & Stable & Polar aprotic \\
$\mathrm{CCl}_{4}$ & 77 & 0.00 & 2.24 & 1.6 & Psi & Yes & Unstable & Nonpolar \\
Water & 100 & & 78.54 & 10.2 & Psi & No & Aggregate & Polar protic \\
DMSO & 189 & 3.96 & 47.2 & 7.2 & Psi & Yes & Stable & Polar aprotic \\
Chloroform & 61 & 1.15 & 4.8 & 4.1 & Psi & Yes & Unstable & Nonpolar \\
Ethyl acetate & 78 & 1.78 & 6.02 & 4.4 & Psi & No & Dispersed & Nonpolar \\
1,4-Dioxane & 101 & 0.45 & 2.3 & 4.8 & Psi & Yes & Stable & Polar aprotic \\
Acetone & 56 & 2.88 & 20.7 & 5.1 & Psi & No & Precipitate & Polar aprotic \\
Cyclohexane & 80 & 0 & 2.28 & 0.2 & Psi & Yes & Unstable & Nonpolar \\
\hline
\end{tabular}

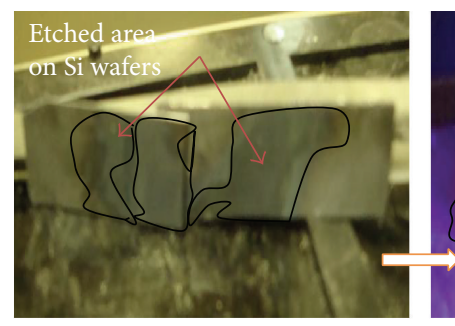

(a)

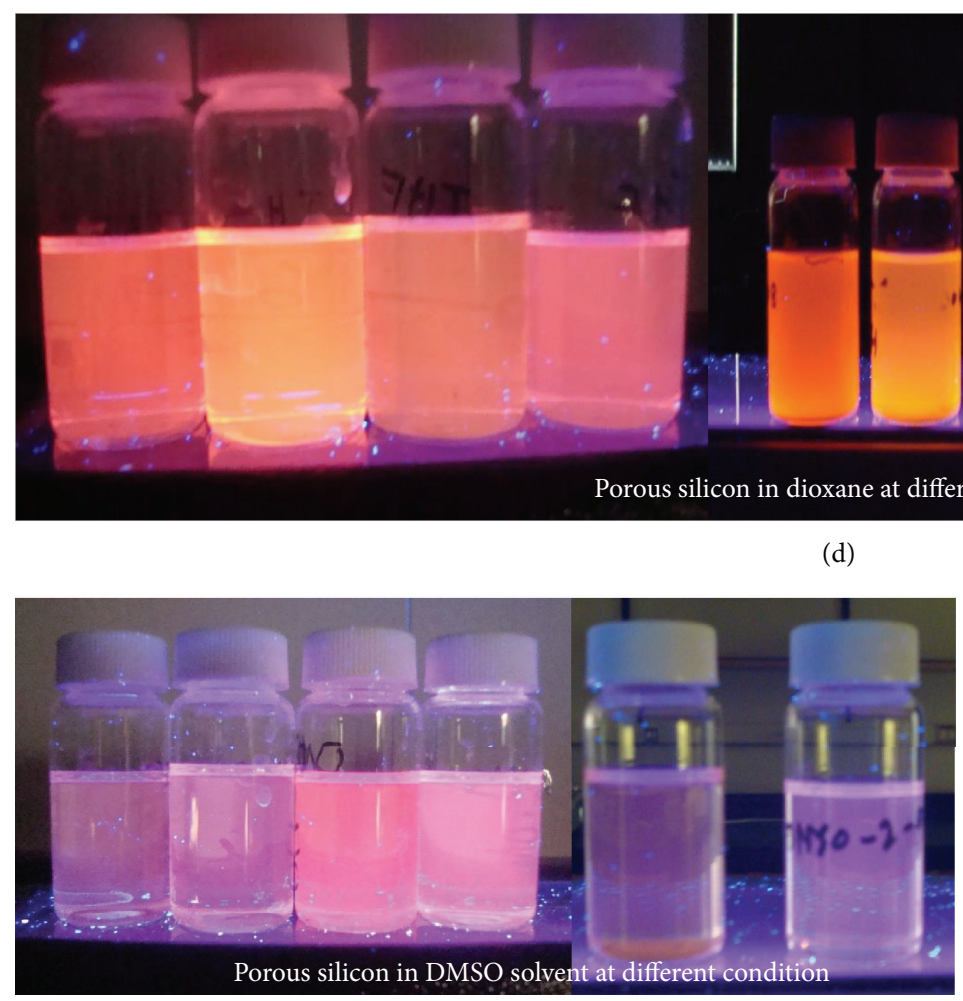

(e)

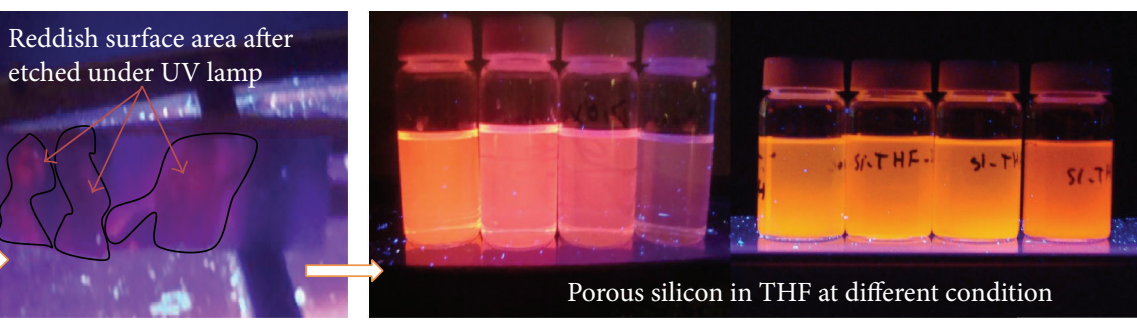

(c)
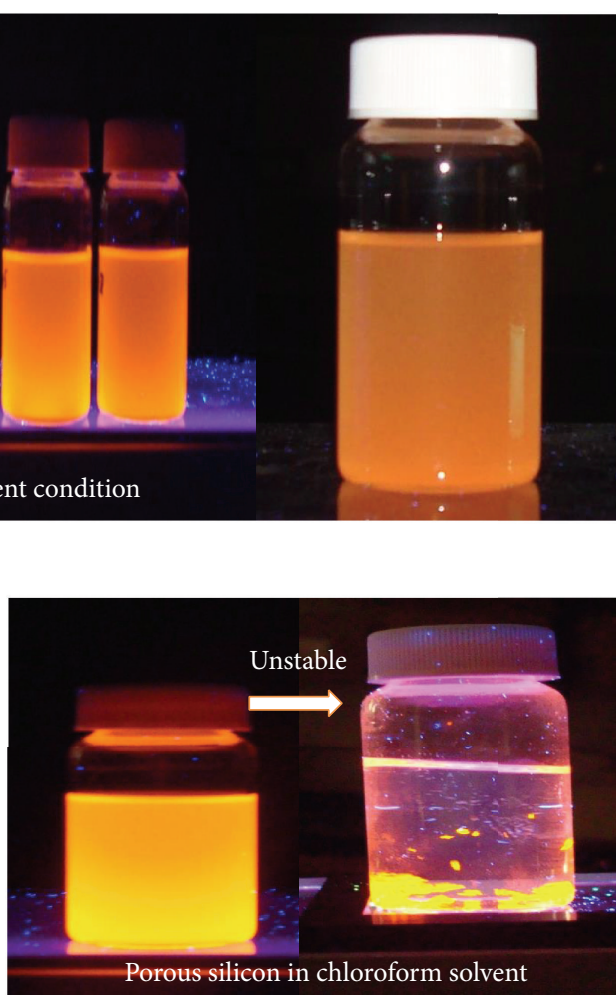

(f)

FIgURE 1: Luminescent light from Psi particles in different solvents under the exposure of UV lamp. 


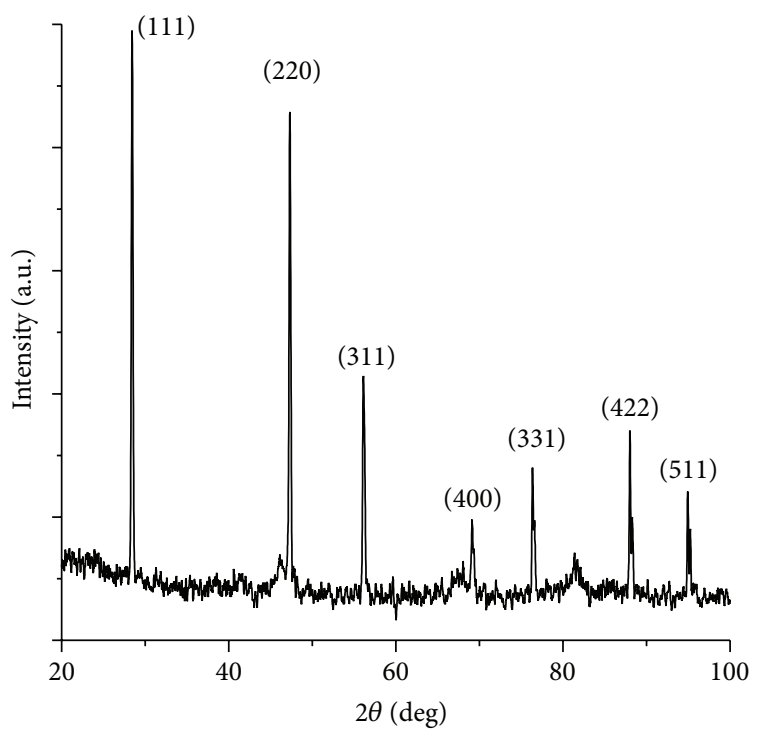

FIGURE 2: XRD pattern of Psi on the etched silicon wafer.

The samples were characterized by FESEM. Figures 3(a)3(c) show the magnification of the plane view of surface morphologies of the Psi in THF, dioxane, and DMSO, respectively. The structure and distribution of Psi in the three solutions are differently observed. For instance, structure of Psi in THF lie in distinct particle to particles whereas Psi based dioxane has a denser structure on the surface as seen in Figures 3(a)-3(c), respectively. Psi in DMSO lies inside layer of solvent. The reason is attributable to the nature of solvent since DMSO has high boiling points that do no dry up. Psi in THF is distinctly oriented whereas Psi has denser structure in dioxane but lies slightly inside the layer of DMSO surface.

Further, TEM analysis was carried out for the determination of size and crystalline nature of Psi in THF, dioxane, and DMSO solution as shown in Figures 4(a)-4(c), respectively, which reveal that most of the Psi were almost spherical in size with average particle size in the range of 5-10 $\mathrm{nm}$. Majority of the Psi in different solutions are larger than $5 \mathrm{~nm}$ in diameter but there is some small size presence less than $5 \mathrm{~nm}$. The crystallites are small dimension which may be the reason for the observed visible photoluminescence. The sizes of Psi in all solvents are almost the same, but distribution of Psi particles in the solvents quite different. For instance, Psi in DMSO is quite monodisperse and Psi in THF and dioxane slightly aggregate which may be due to nature solvents. The evaluated particles size from the XRD pattern is in good agreement with the TEM $(10 \mathrm{~nm})$ results. TEM lattice images show nice crystalline and clear lattice fringes of a single nanocrystal. In Figures 5(a)-5(c), the corresponding selected area electron diffraction (SAED) pattern indicates the crystallinity and preferential orientation of the Si particles without any additional diffraction spots of THF, dioxane, and DMSO particles and which is in good agreement with the hexagonal structure of the XRD results. The patterns show strong crystalline dots but some weak diffused crystalline rings exist in all images. The diffused weak rings of the Psi are clearer in THF and DMSO than in dioxane solvent.
TABLE 2: Absorption peaks of Psi in THF, dioxane, and DMSO.

\begin{tabular}{lccc}
\hline Psi in solvents & $\begin{array}{c}\text { Number of wafer piece } \\
(\text { Pcs }) \\
(1 \times 4 \mathrm{~cm}) / \text { Pcs }\end{array}$ & $\begin{array}{c}\text { Absorption } \\
\text { peaks }\end{array}$ & Absorbance \\
\hline \multirow{2}{*}{ THF } & 4 & $250 \mathrm{~nm}$ & Strong \\
& & $320 \mathrm{~nm}$ & Strong \\
THF & 6 & $240 \mathrm{~nm}$ & Shoulder \\
& 9 & $268 \mathrm{~nm}$ & Strong \\
THF & 4 & $288 \mathrm{~nm}$ & Shoulder \\
& & $223 \mathrm{~nm}$ & Strong \\
1,4-Dioxane & 6 & $322 \mathrm{~nm}$ & Medium \\
& & $232 \mathrm{~nm}$ & Strong \\
1,4-Dioxane & & $314 \mathrm{~nm}$ & Weak \\
& 9 & $235 \mathrm{~nm}$ & Strong \\
1,4-Dioxane & & $270 \mathrm{~nm}$ & Shoulder \\
& 4 & $267 \mathrm{~nm}$ & Strong \\
DMSO & & $313 \mathrm{~nm}$ & split \\
& & $272 \mathrm{~nm}$ & Strong \\
DMSO & 6 & $265 \mathrm{~nm}$ & Strong \\
DMSO & 9 & &
\end{tabular}

The elemental analysis of the Psi in the solvents was performed by EDAX spectra suggest that the presence of Psi. The spectra of the Psi in the solvents confirmed that no peaks other than for the elements $\mathrm{Si}$ were observed as shown in Figures 6(a)-6(c), besides $\mathrm{Cu}$ and $\mathrm{C}$ peaks from carboncoated $\mathrm{Cu}$ grid used in TEM, indicating the purity level of the silicon materials.

The absorption spectra of Psi in THF, dioxane, and DMSO were studied at quantity of $\mathrm{Si}$ wafer pieces $(4,6$, and 9) as shown in Figure 7. Effects on the absorption peaks of different quantity based Psi in THF are observed but bandwidth lies within the same spectral region. The effects on peaks and bands shape with respect to quantity of wafers may correspond to the change in densities of Psi within THF environment as shown in Figure 7(a). There is also influence in relative absorbance in accordance with the increase of quantity of wafer piece. Psi in dioxane based solution shows greater in absorbance at 6 Pcs than 9Pcs of silicon wafer as shown in Figure 7(b). Peak positions and bandwidths of three Psi solutions are influenced due to different quantity of wafers. As seen in Figure 7(c), absorption peaks of Psi in DMSO solution observed same behavior as the Psi in THF that higher quantity of wafer piece shows greater in absorbance. But the correspondence peaks of Psi in THF and dioxane become merging as observed in DMSO at higher quantity of wafer piece. Comparison of absorption peaks position of the Psi in three solutions at the same quantity of wafer piece shows that the correspondence peaks and intensities are influenced as listed in Table 2. It means that peak positions of Psi do not depend on quantity of Si wafer piece. Moreover, it may mention that density or concentration of porous silicon particles in solution may possibly depend on the cross-section of etched area on silicon wafer as seen in Figure 1 ((a)-(b), insect circle area). The etched surface area may vary wafer piece to piece because of the nonuniformity etching in HF based etching solution. Larger etched area of 


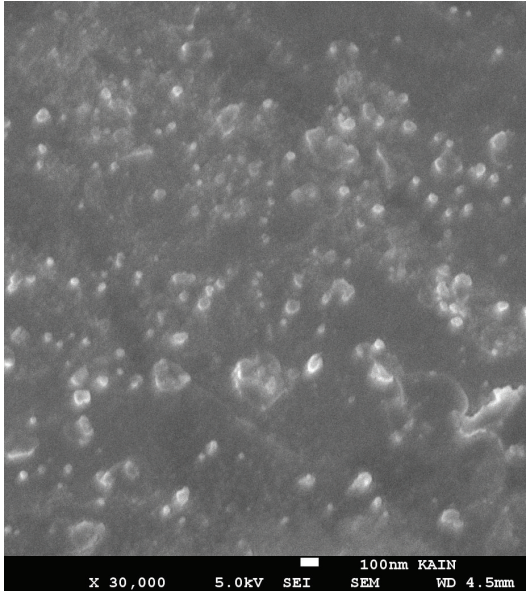

(a)

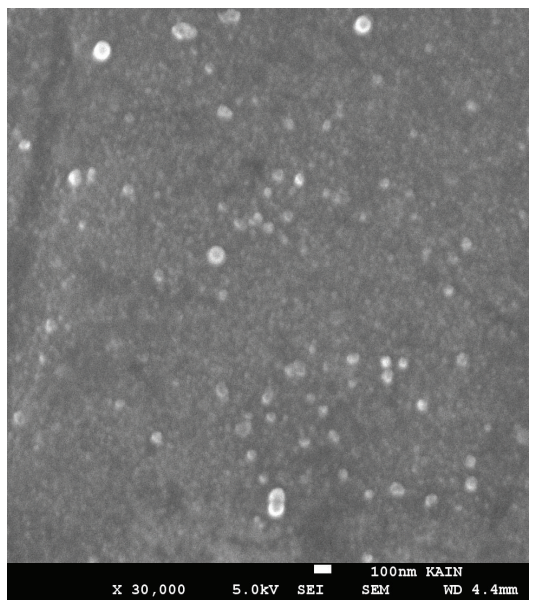

(b)

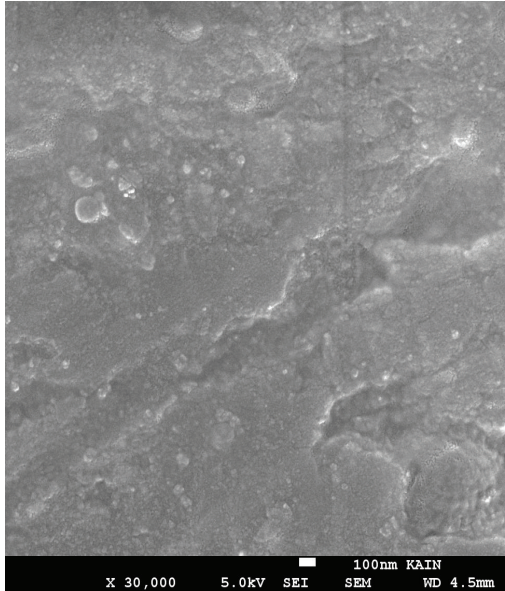

(c)

FIgure 3: SEM images of Psi in (a) THF, (b) dioxane, and (c) DMSO solvent.

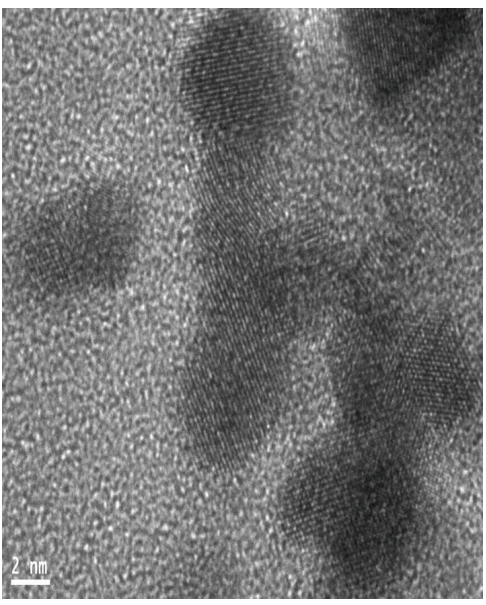

(a)

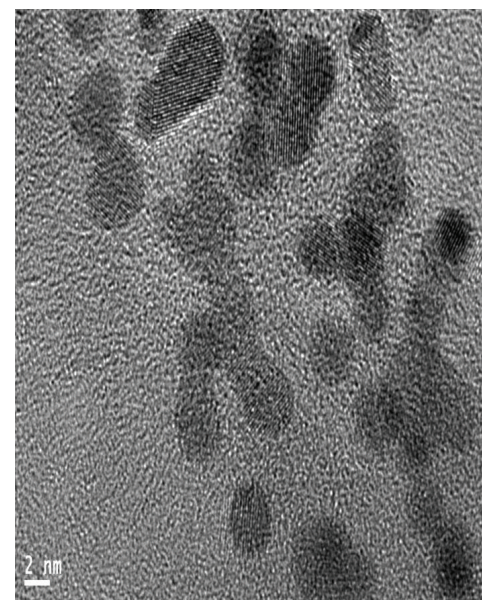

(b)

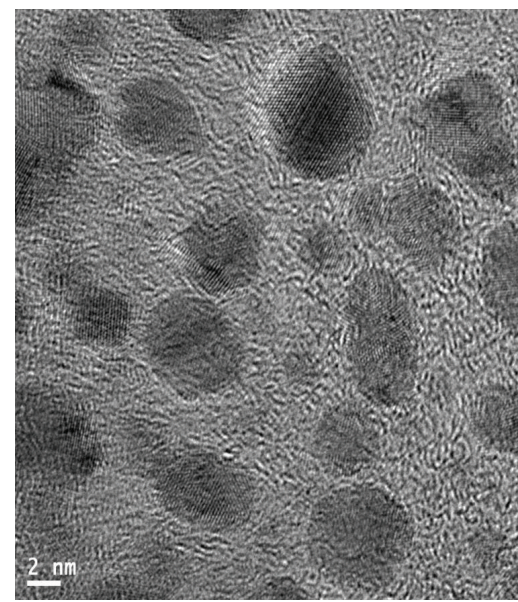

(c)

FIgURE 4: HRTEM images of Psi in (a) THF, (b) dioxane, and (c) DMSO solvents.

wafers may produce greater quantity of particles in solvents. It may be a reason that absorbance of Psi in dioxane solution at higher quantity of $\mathrm{Si}$ wafer decreases.

On the other hand, the absorption edges in all cases shift towards lower wavelength region as the number of wafer piece decreases. The blueshift is attributed to the BursteinMoss effect. Moreover, the motion of fermi level into the conduction band may influence to absorption edges when the synthesing condition is changed. This phenomenon can also be observed clearly in Figure 8 which shows the relationship between absorption coefficients as a function of photon energy. The absorption coefficient $(\alpha)$ can be calculated from the following relation:

$$
\alpha=\frac{A}{d},
$$

where $A$ is the absorbance of the samples and $d$ is the thickness.
The optical band gap of the films is determined by applying the Tauc model [30] and the Davis and Mott model [31] in the high absorbance region:

$$
(\alpha h v)=C\left(h \nu-E_{g}\right)^{n}
$$

where $h v$ is the photon energy, $E_{g}$ is the optical band gap, $C$ is a constant, and $n$ is a parameter that characterizes the optical absorption process and theoretically is equal to $1 / 2$ and 2 for direct allowed and indirect allowed transitions, respectively [31]. In order to find the band gap of samples, the relationship between $(\alpha h v)^{1 / 2}$ and $h v$ is plotted in Figures 8(a)-8(c). The value of band gap can be obtained by extrapolating the linear portion of the curve to the photon energy axis and the values are listed in Table 3. As seen in Table 3, optical gap of Psi in THF, dioxane, and DMSO at different quantity of wafer Si piece $(4,6$, and 9$)$ are varied due to the effect of densities of porous silicon in solvents and due to different densities of 


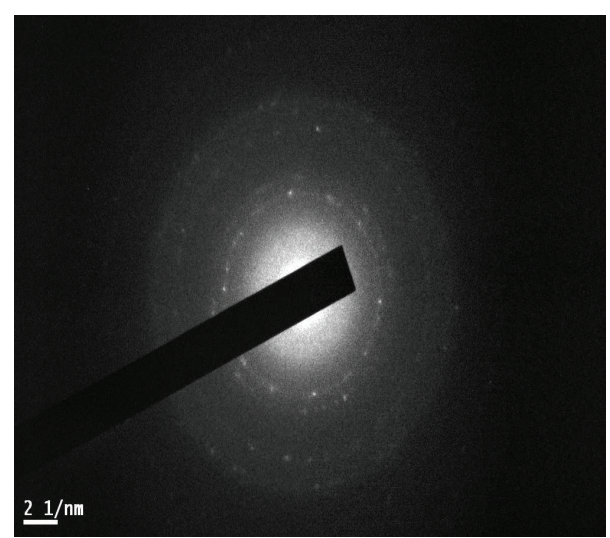

(a)

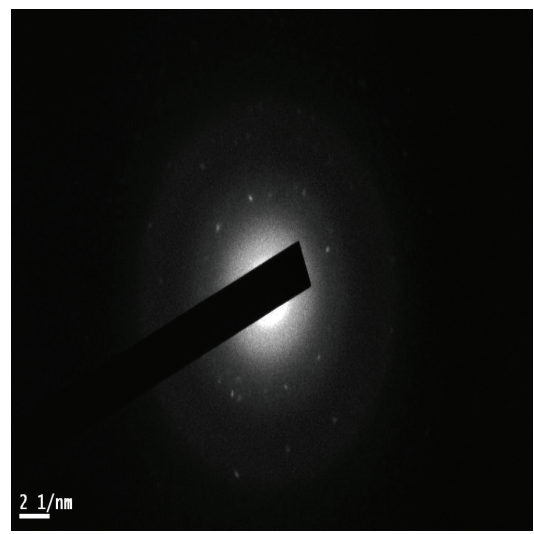

(b)

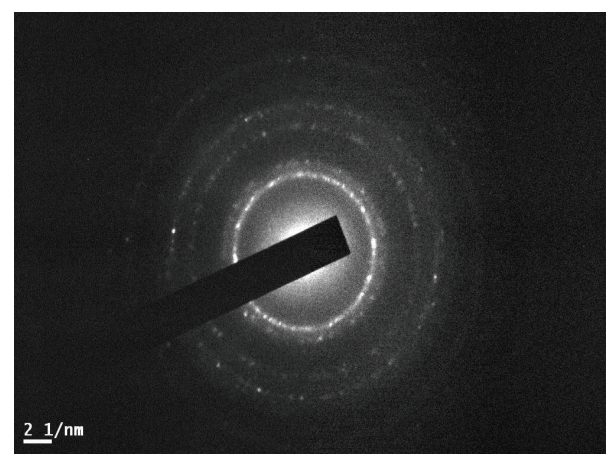

(c)

FIGURE 5: Electron diffraction pattern of Psi in (a) THF, (b) dioxane, (c) DMSO solvent solvents.

particles which may influence to absorption edges of Psi in solvents.

In view of the importance to photoluminescence properties, emission spectra of Psi in THF, dioxane, and DMSO at 4, 6 , and 9 quantity of wafer piece were studied. The emission intensity of Psi in all solvents is pronounced greater at the $355 \mathrm{~nm}$ excitation wavelength. Therefore, $355 \mathrm{~nm}$ excitation wavelength was selected for all samples in order to understand the PL behavior of Psi corresponding to the quantity of wafer piece. Recently our group reported that the emission spectra of Psi in THF and dioxane using 4 wafer pieces also
TABLE 3: List of the Eg values of Psi in different solvents.

\begin{tabular}{lc}
\hline Sample & Energy band gap (Eg) \\
\hline Psi in THF (4 Pcs wafer) & $3.04 \mathrm{eV}$ \\
Psi in THF (6 Pcs wafer) & $2.57 \mathrm{eV}$ \\
Psi in THF (9 Pcs wafer) & $2.82 \mathrm{eV}$ \\
Psi in dioxane (4 pcs wafer) & $2.73 \mathrm{eV}$ \\
Psi in dioxane (6 pcs wafer) & $2.50 \mathrm{eV}$ \\
Psi in dioxane (9 pcs wafer) & $2.63 \mathrm{eV}$ \\
Psi in DMSO (4 Pcs wafer) & $2.27 \mathrm{eV}$ \\
Psi in DMSO (6 Pcs wafer) & $2.43 \mathrm{eV}$ \\
Psi in DMSO (9 Pcs wafer) & $2.50 \mathrm{eV}$ \\
\hline
\end{tabular}

incorporated in sol gel host $[22,23]$. Weak emissions lights were obtained from the Psi doped sol gel prepared using 4 quantities of silicon wafer piece. Therefore, by quantifying the wafer piece in etching process, emission properties of Psi in solvents were studied. For instance, emission peaks of THF varied in accordance with quantity of wafer pieces. The emission peak of Psi in THF at different excitation wavelength is seen in Figures 9(a)-9(b). It is observed that intensities of the Psi depend on the excitation energy. As an example, $355 \mathrm{~nm}$ excitation of the samples exhibits stronger emission as compared to the 265 and $365 \mathrm{~nm}$ excitation. There is influenced in emission peak positions of Psi in THF at 4, 6, and 9 quantity of Si wafer piece. The corresponding peaks lie at $633 \mathrm{~nm}$ (4 Pcs), $619 \mathrm{~nm}$ (6 Pcs), and $620 \mathrm{~nm}$ (9Pcs), respectively. The emission and excitation of Psi dispersed dioxane at the same condition as in THF was taken and found to be same characteristic in peak and intensities. As shown in Figures 9(c)-9(d), intensity of emission peak depends on the excitation wavelengths. The emission and excitation spectra of Psi dioxane at 4,6, and 9 silicon wafer piece are shown in Figures 9(c)-9(d). The corresponding peaks lie at $628 \mathrm{~nm}$ of (4 Pcs), $617 \mathrm{~nm}$ (6 Pcs), and $632 \mathrm{~nm}$ (9Pcs), respectively. Similarly, emission and excitation peaks of Psi in DMSO solvent are shown in Figures 9(e)-9(f). Effect in emission intensity of Psi DMSO is observed due to excitation energy. The emission intensity is stronger at the $355 \mathrm{~nm}$ excitation. The observed emission and excitation peaks of Psi DMSO are stronger at $355 \mathrm{~nm}$ excitation wavelength and corresponding the positions at $625 \mathrm{~nm}$ (4 Pcs), $636 \mathrm{~nm}(6 \mathrm{Pcs})$, and $629 \mathrm{~nm}$ (9 Pcs), respectively. The comparison data on emission and excitation peaks of Psi in THF, dioxane, and DMSO are listed in Table 4. As seen in present results, intensities of Psi particles in solvents possibility depend on the nature of solvents and the cross-section area of etching. Higher cross-section area of etching on wafer surface can produce greater quantity of silicon particles in solvents. In present observation shows that there is influenced in emission intensity due to more quantity of Psi in solution.

Moreover, it seems that photoluminescence intensity depends on the Psi distribution containing in solvents and nature of solvents. Although the reason of nonluminescent Psi in some solvents is not clear but it may corelate to dielectric constant of solvents as seen in Table 1. Smaller values of dielectric constant in solvents are obtained in $\mathrm{PL}$ 
TABLE 4: Emission and excitation peak positions of Psi in THF, dioxane, and DMSO.

Emission spectra of Psi in solvents

Number of silicon wafer piece (Pcs) Excitation wavelength (nm) Emission peaks (nm) Excitation peaks (nm)

\begin{tabular}{|c|c|c|c|c|}
\hline \multirow{3}{*}{ THF } & 4 & 265 & 633 & 303 \\
\hline & & 355 & & \\
\hline & & 365 & & \\
\hline \multirow{2}{*}{ THF } & 6 & 355 & 619 & 349 \\
\hline & 9 & 355 & 620 & 345 \\
\hline \multirow{2}{*}{ Dioxane } & 4 & 265 & 628 & 335 \\
\hline & & 355 & & \\
\hline \multirow{2}{*}{ Dioxane } & 6 & 355 & 617 & 352 \\
\hline & 9 & 355 & 632 & 345 \\
\hline \multirow{3}{*}{ DMSO } & 4 & 355 & 625 & \\
\hline & 6 & 355 & 636 & 349 \\
\hline & 9 & 355 & 629 & 348 \\
\hline
\end{tabular}

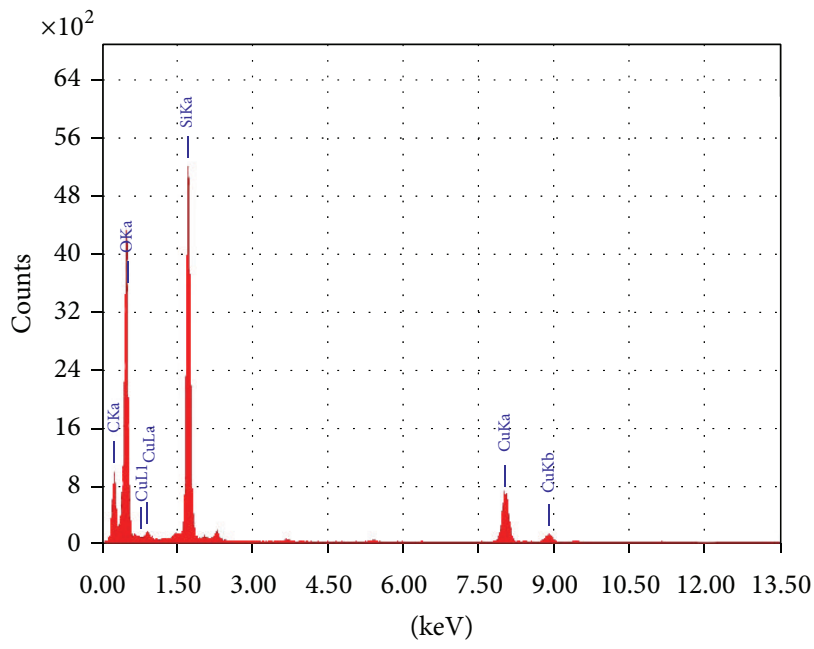

(a)

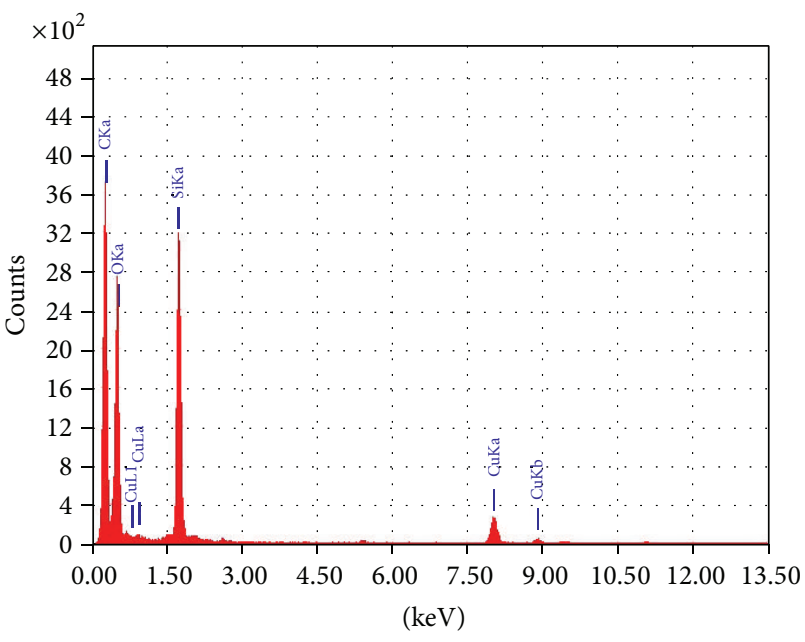

(b)

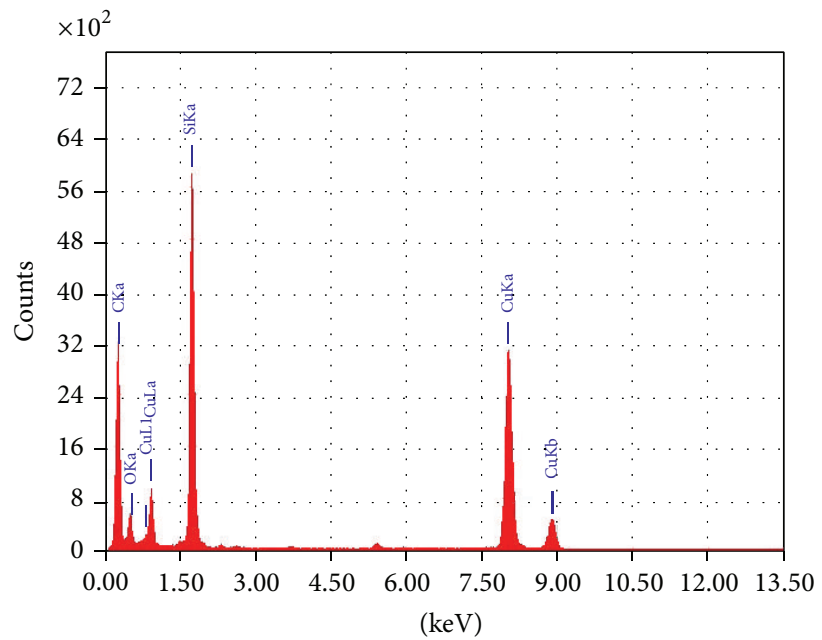

(c)

Figure 6: EDAX of Psi in (a) THF, (b) dioxane, and (c) DMSO solvents. 


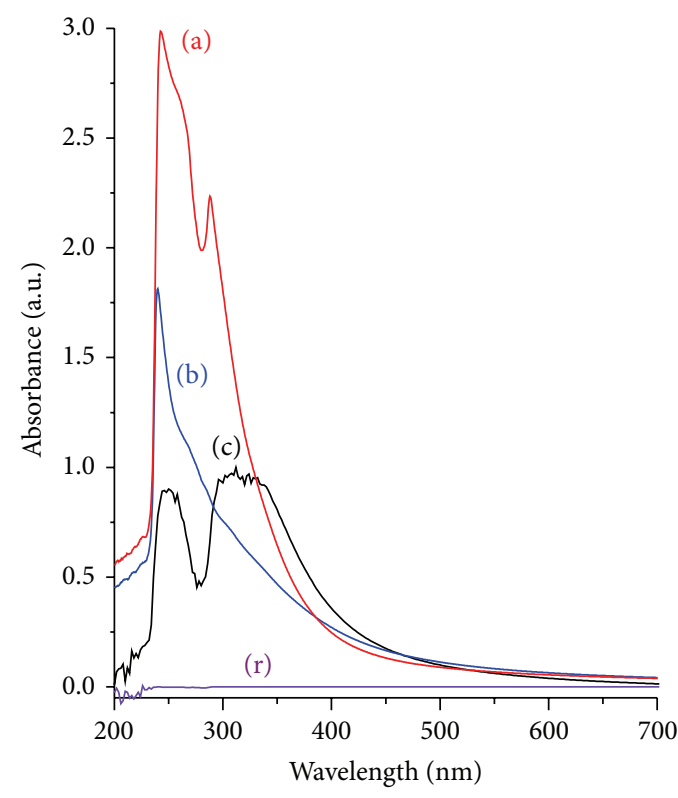

(a): 9 Pcs $(1 \times 4 \mathrm{~cm})$ in THF solvent

(b): 6 Pcs

(c): 4 Pcs

(r): THF (ref.)

(a)

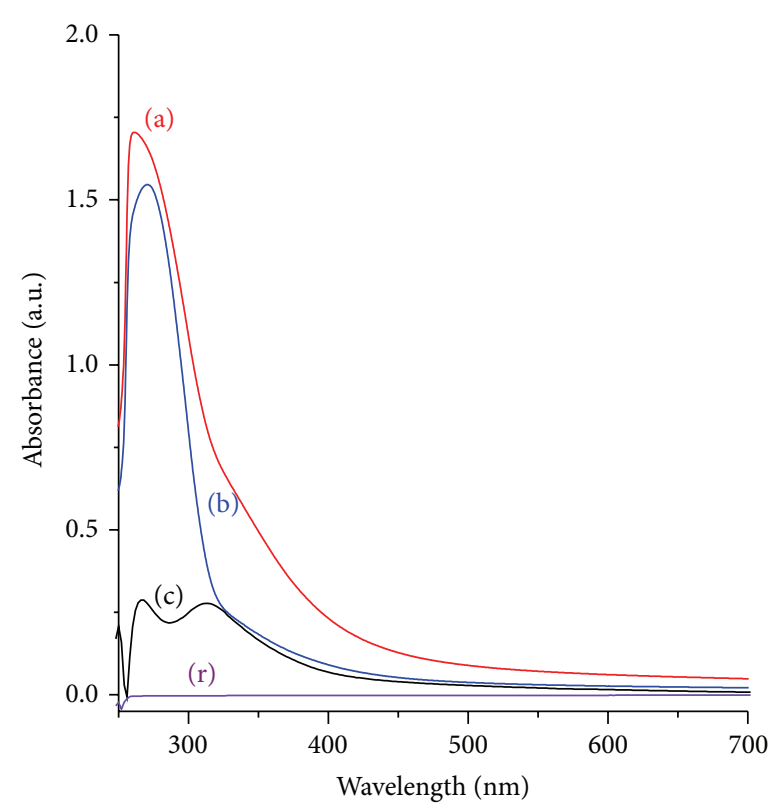

(a): 9 Pcs $(1 \times 4 \mathrm{~cm})$ in DMSO solvent

(b): 6 Pcs

(c): 4 Pcs

(r): DMSO solvent (ref.)

(c)

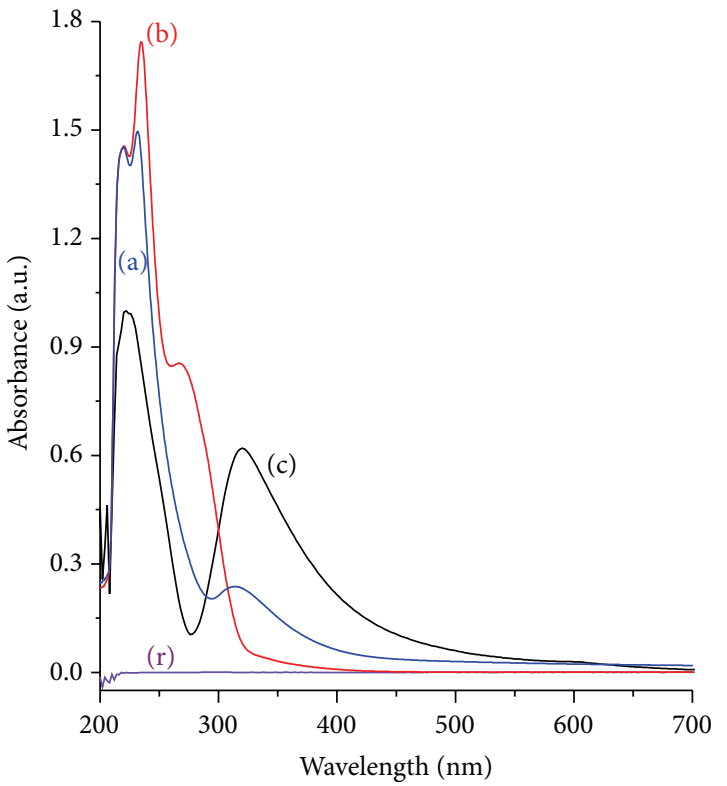

(a): 9 Pcs $(1 \times 4 \mathrm{~cm})$ in dioxane

(b): 6 Pcs

(c): 4 Pcs

(r): dioxane (ref.)

(b) 


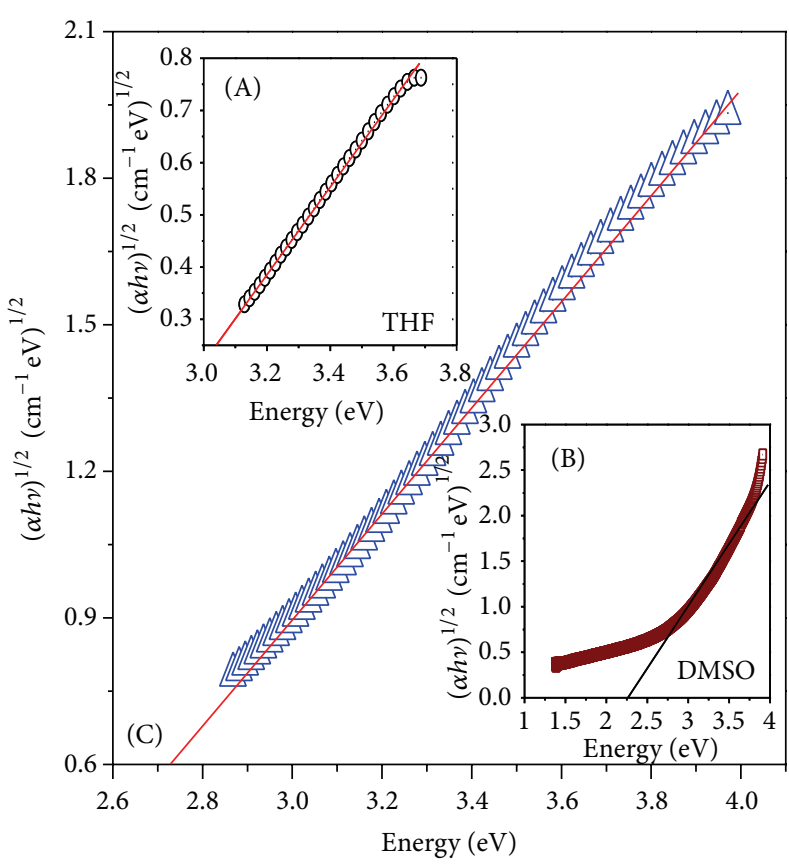

$\triangle$ 4-Dioxane

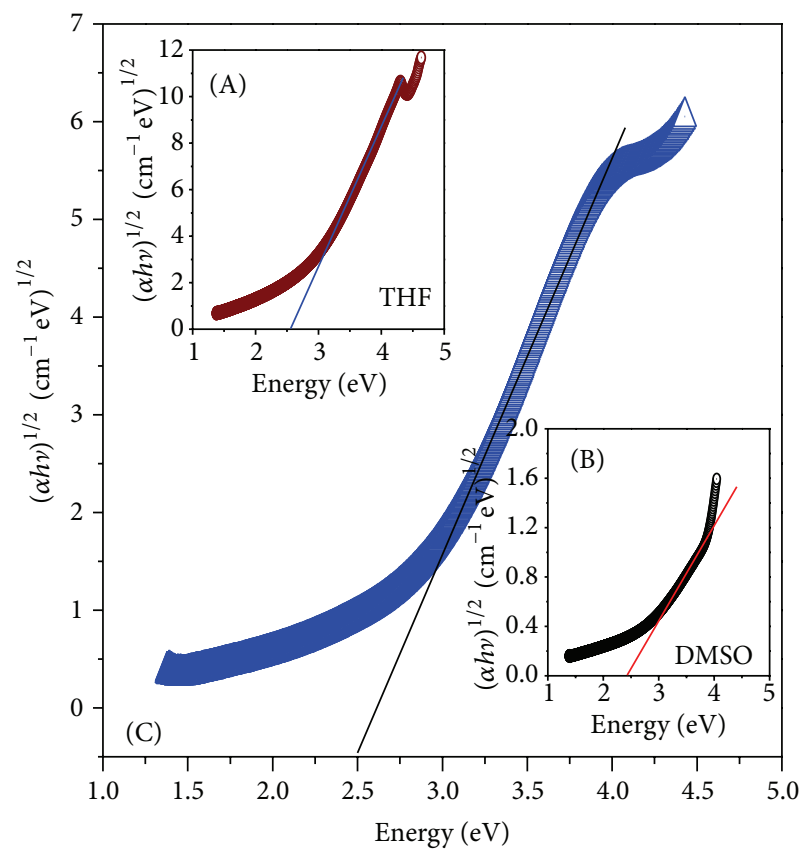

$\triangle$ 6-Dioxane

(a)

(b)

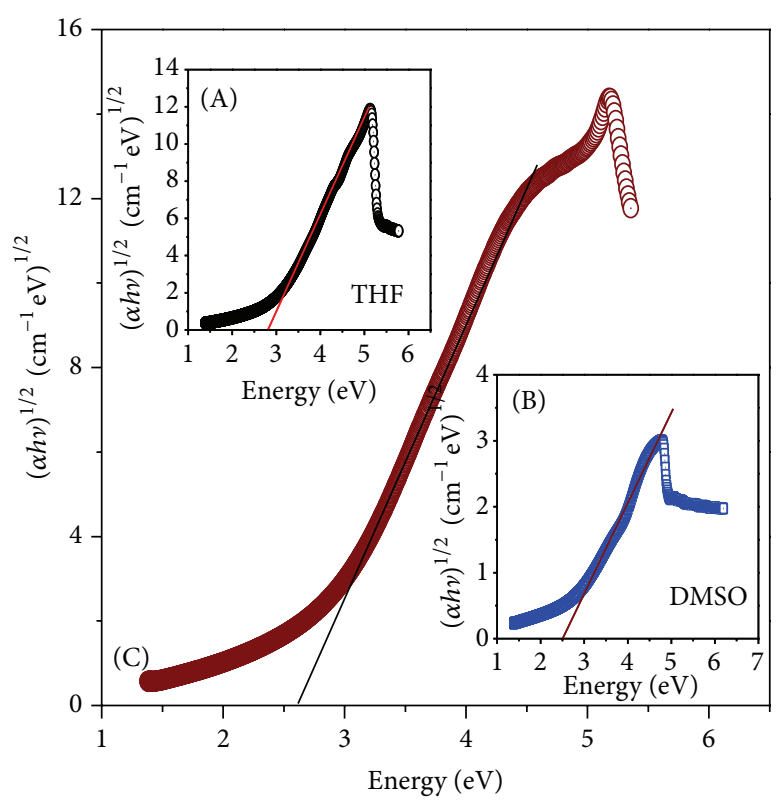

$\odot$ 9-Dioxane

(c)

Figure 8: Energy band gap (Eg) of Psi in THF, dioxane, and DMSO solvents at different wafer piece.

except DMSO which has a large dielectric value. Our results may correspond to previous work [32] that for values of the dielectric constant larger than 20, the Onsager length becomes of the order of the nanostructure size, and no further quenching is observed. Therefore, the luminescent of colloidal Psi solutions are in aprotic solvents. It may also be possible that those solvents do not contain an $\mathrm{O}-\mathrm{H}$ bond which helps to emit light of Psi in solvent with stability since aprotic solvents do not contain such bonds. No doubt that PL of Psi in some nonpolar solvents are exhibited and welldispersed but not stable as listed in Table 1.

In addition, thermal effect on PL properties of a Psi colloidal solution of each solvent was studied. Temperature annealing of the samples was conducted at $25-75^{\circ} \mathrm{C}$ in range 


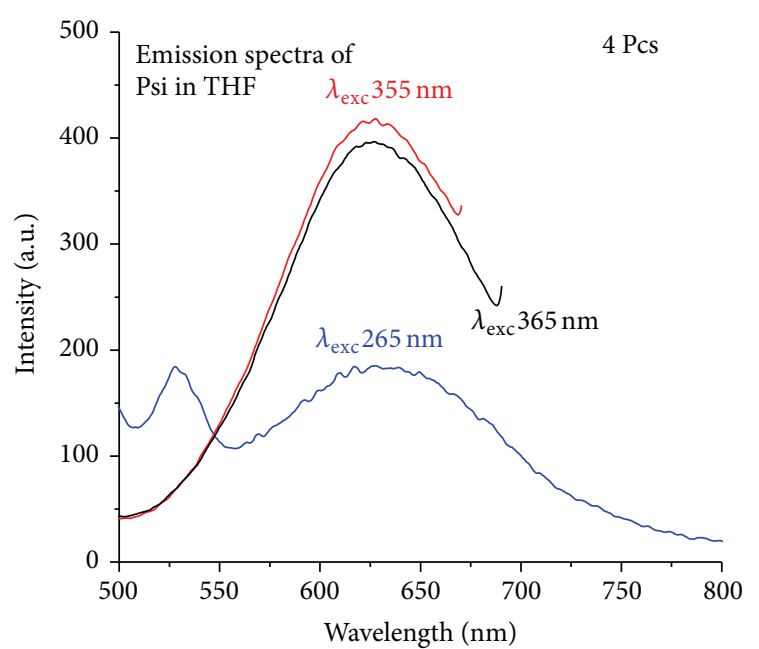

(a)

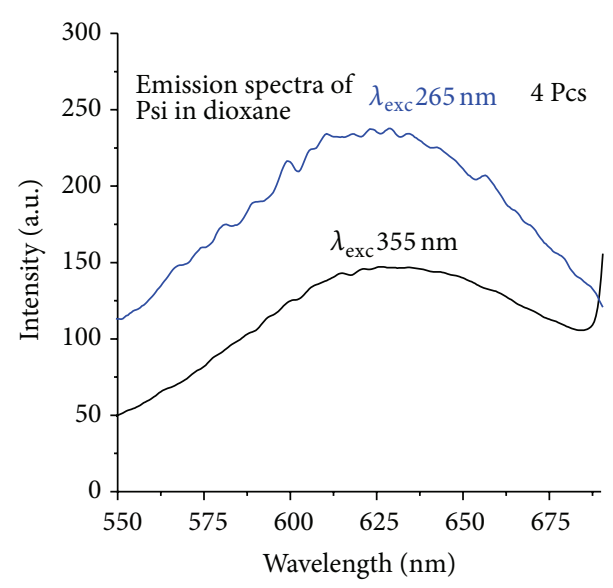

(c)

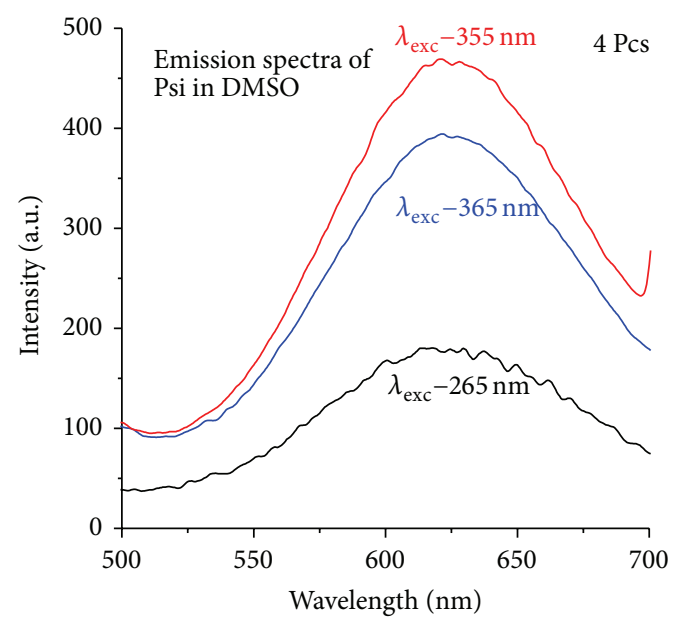

(e)

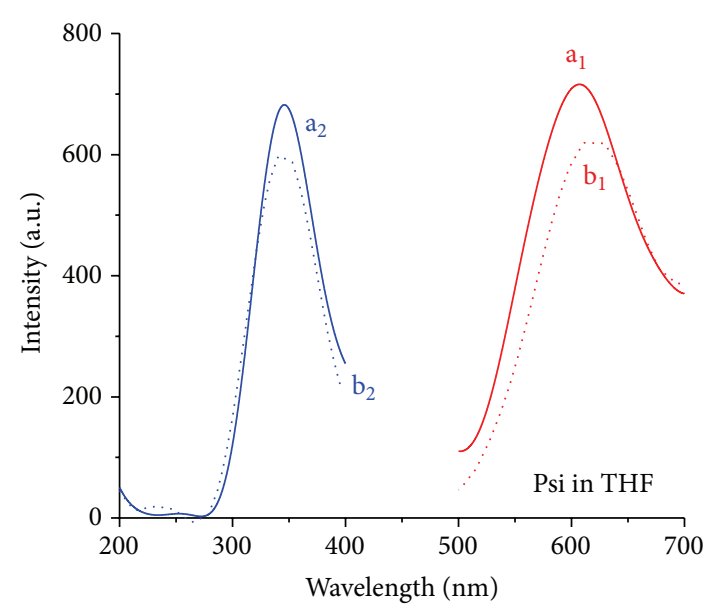

(b)

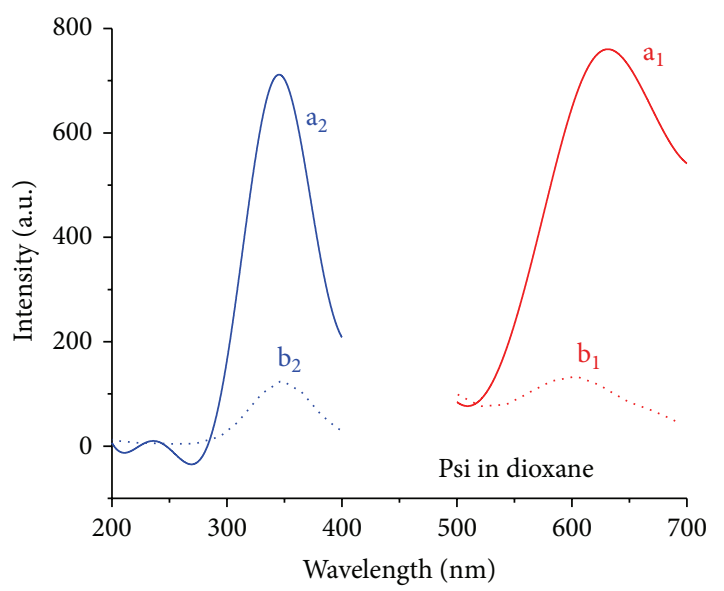

(d)

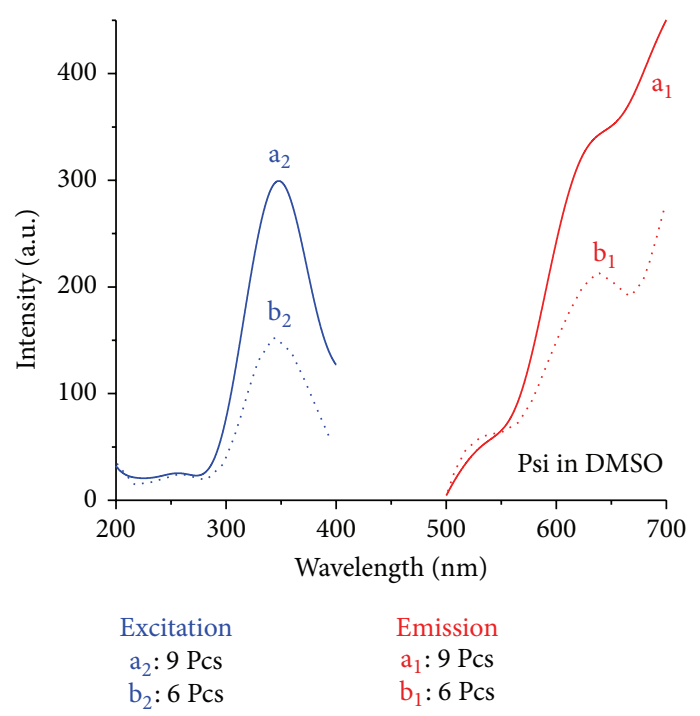

(f)

FIGURE 9: Emission and excitation spectra of Psi in (a and b) THF, (c and d) dioxane, and (e and f) DMSO. 


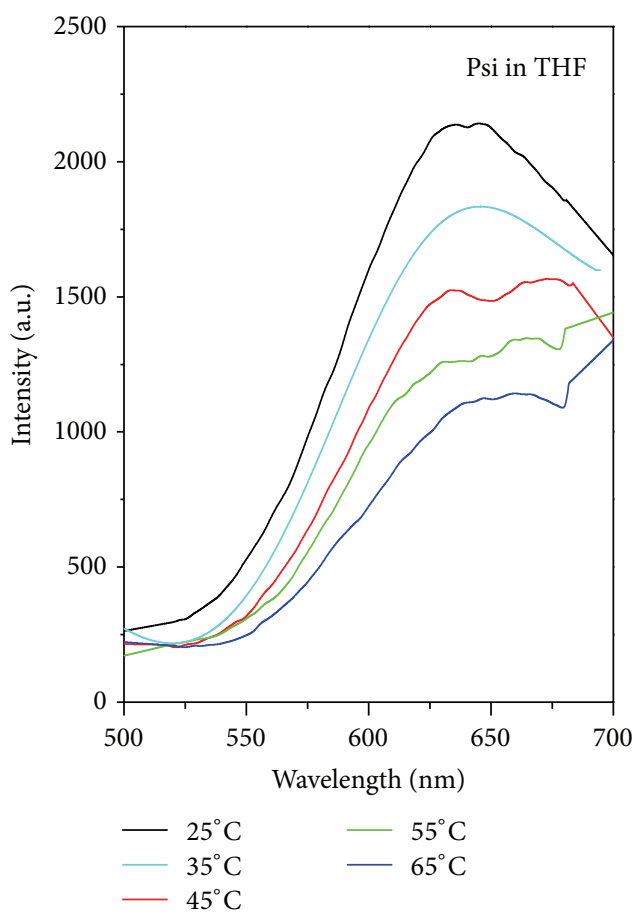

(a)

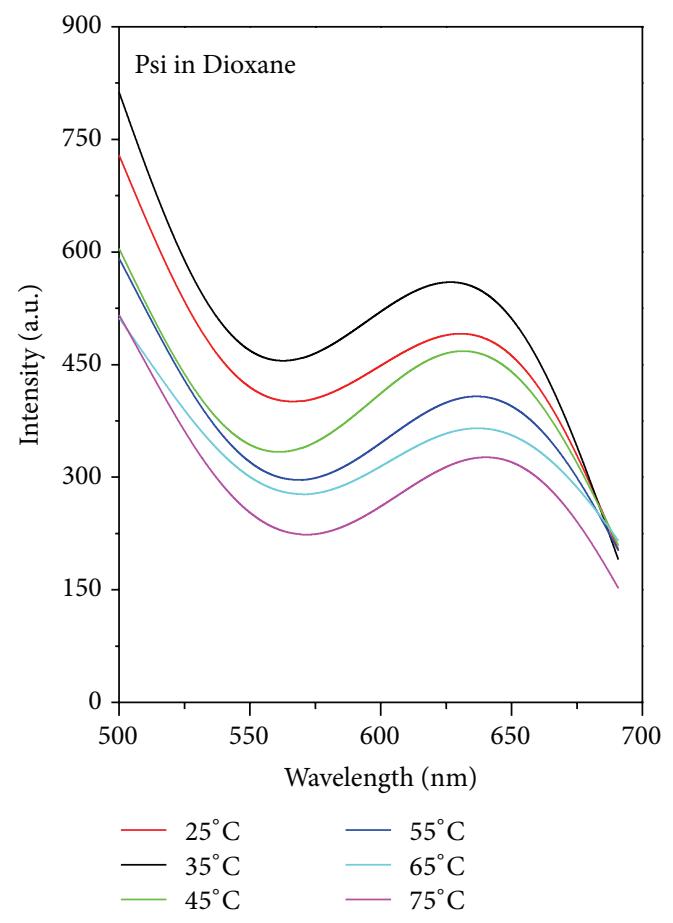

(b)

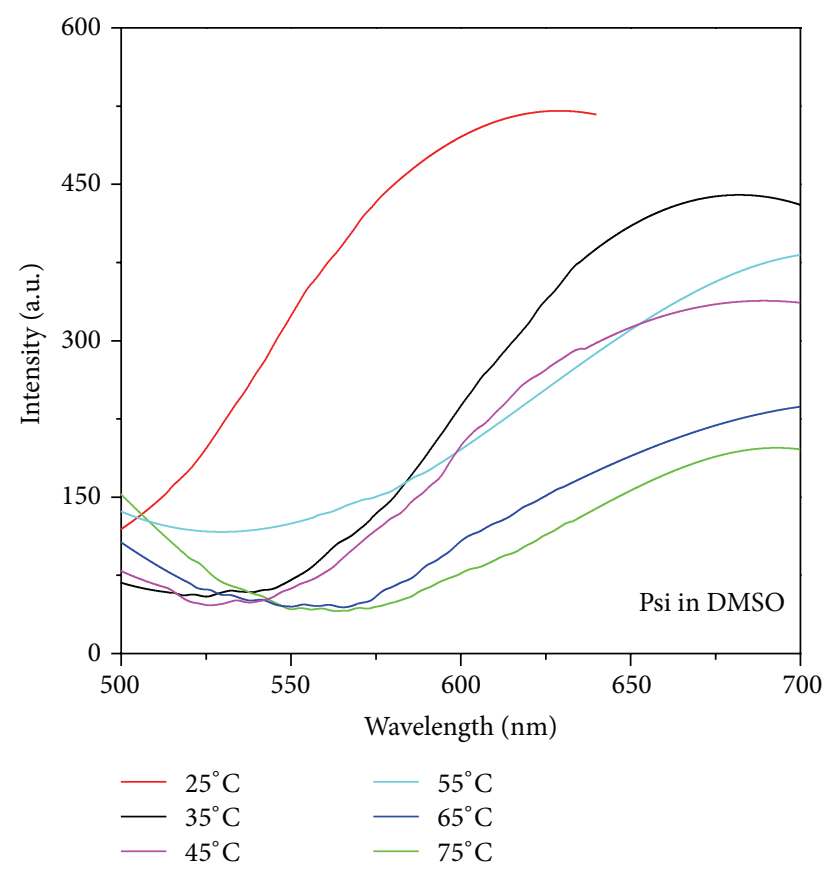

(c)

Figure 10: Annealing effect on emission spectra of Psi in (a) THF, (b) dioxane, and (c) DMSO.

of temperature. The emission feature of Psi THF solution annealed from 25 to $65^{\circ} \mathrm{C}$ is shown in Figure 10 (a). The intensity of the Psi samples linearly decays till $65^{\circ} \mathrm{C}$ and then non-uniformity in peaks observed due to evaporation of solution at higher temperature. Although the intensity of Psi dioxane decreases with respect to temperature, shape of peaks is uniformed up to $75^{\circ} \mathrm{C}$ as shown in Figure 10(b). However, behavior of Psi in DMSO under thermal alters from the THF and dioxane based solution, as seen in Figure 10(c). The emission spectra of Psi dioxane is observed as curve shape till $45^{\circ} \mathrm{C}$ then slightly become linear. The intensity reduces with temperature but peak at $55^{\circ} \mathrm{C}$ induces higher. It may mention that peak positions of THF solution under thermal annealing is more affected than the dioxane and DMSO as listed in 

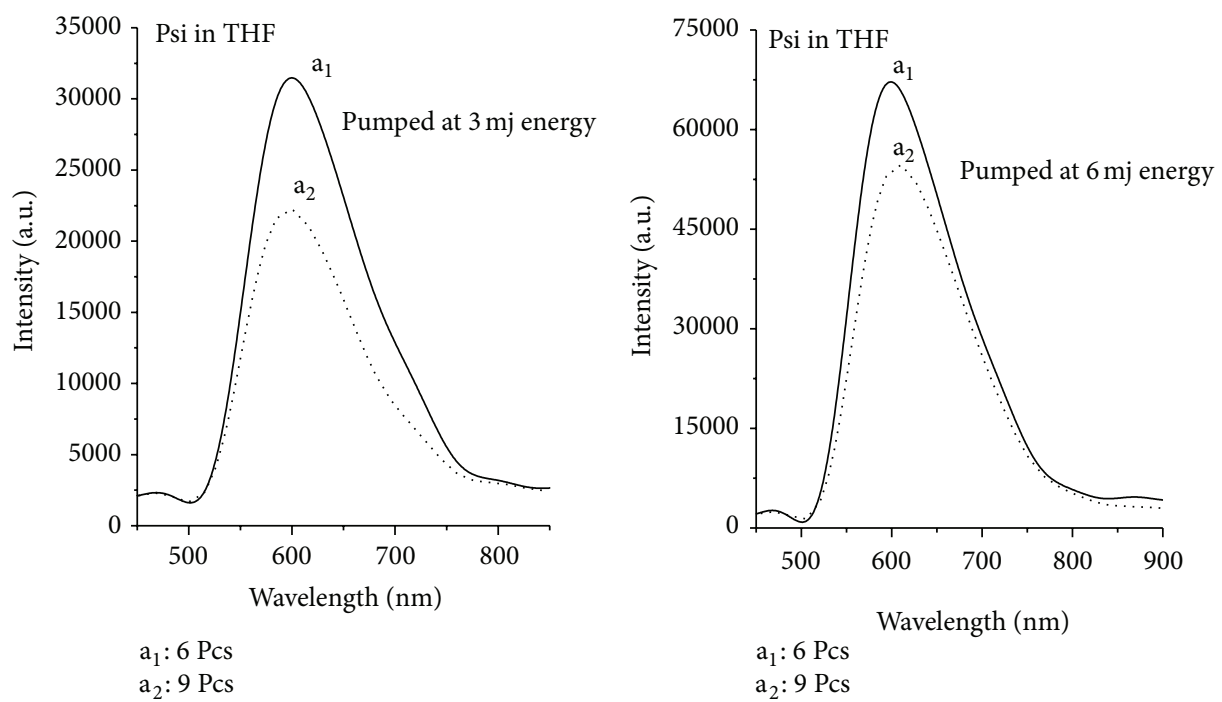

(a)

(b)
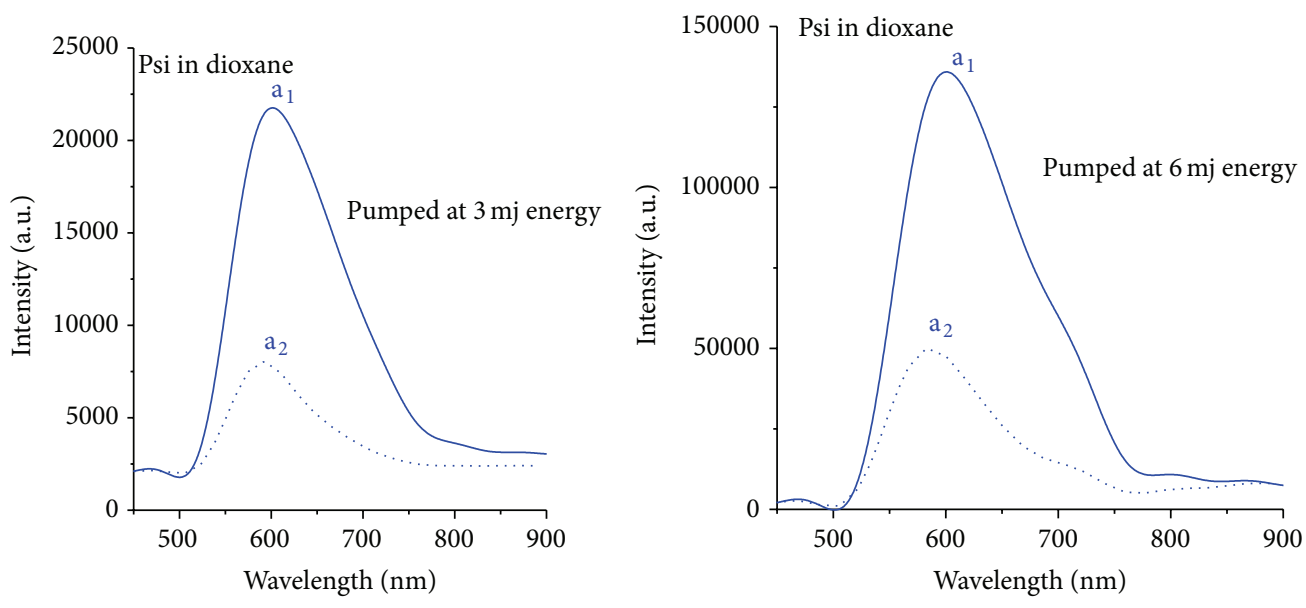

$$
\begin{array}{ll}
a_{1}: 9 \text { Pcs } & a_{1}: 9 \text { Pcs } \\
a_{2}: 6 \text { Pcs } & a_{2}: 6 \text { Pcs }
\end{array}
$$

(c)

(d)
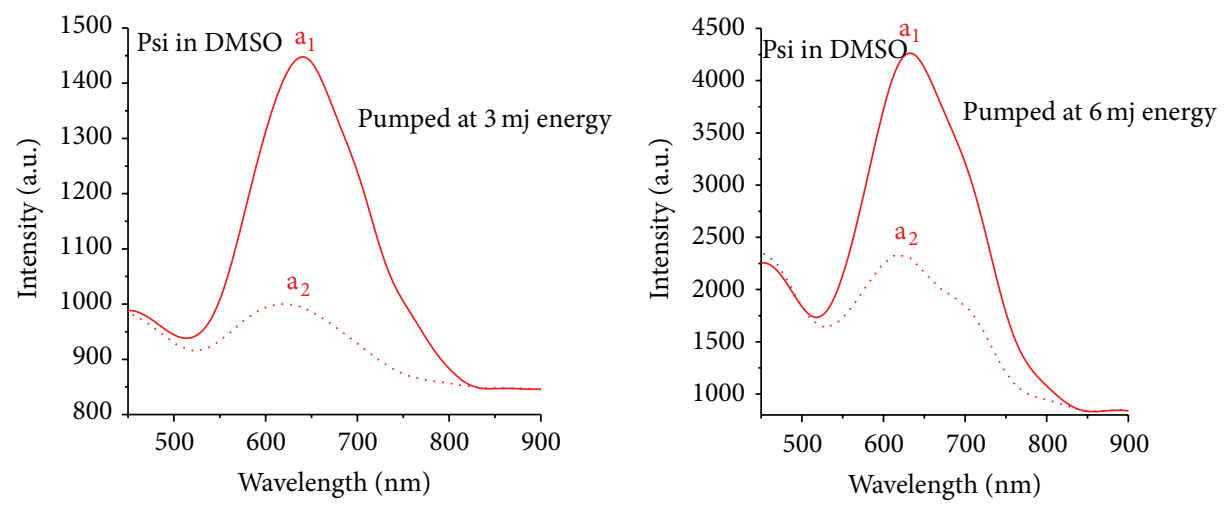

$a_{1}: 9$ Pcs
$a_{2}: 6$ Pcs

$a_{1}: 9$ Pcs

$\mathrm{a}_{2}: 6$ Pcs

(e)

(f)

FIgURE 11: Spontaneous spectra of Psi in (a) THF, (b) dioxane, and (c) DMSO under Pico second $355 \mathrm{~nm}$ laser at 3 and 6 mj energy. 
TABLE 5: Emission peak positions of Psi in THF, dioxane, and DMSO under different temperature.

\begin{tabular}{lccc}
\hline Temperature & $\begin{array}{c}\text { Emission peaks } \\
\text { of Psi in THF }\end{array}$ & $\begin{array}{c}\text { Emission peaks } \\
\text { of Psi dioxane }\end{array}$ & $\begin{array}{c}\text { Emission peaks } \\
\text { of Psi in DMSO }\end{array}$ \\
\hline $25^{\circ} \mathrm{C}$ & $637 \mathrm{~nm}$ & $633 \mathrm{~nm}$ & $675 \mathrm{~nm}$ \\
$35^{\circ} \mathrm{C}$ & $643 \mathrm{~nm}$ & $637 \mathrm{~nm}$ & $675 \mathrm{~nm}$ \\
$45^{\circ} \mathrm{C}$ & $648 \mathrm{~nm}$ & $639 \mathrm{~nm}$ & $677 \mathrm{~nm}$ \\
$55^{\circ} \mathrm{C}$ & $655 \mathrm{~nm}$ & $643 \mathrm{~nm}$ & $677 \mathrm{~nm}$ \\
$65^{\circ} \mathrm{C}$ & $655 \mathrm{~nm}$ & $643 \mathrm{~nm}$ & $679 \mathrm{~nm}$ \\
$75^{\circ} \mathrm{C}$ & Evaporated & $645 \mathrm{~nm}$ & $681 \mathrm{~nm}$ \\
\hline
\end{tabular}

Table 5. Overall the effects on PL of Psi solutions seem to be depending on the nature solvents. For instance, boiling point of DMSO is different from dioxane and THF. The evaporation rate of THF is higher than that of DMSO and dioxane.

Finally, spontaneous light from Psi colloidal solutions were studied under high Pico second $355 \mathrm{~nm}$ laser source in the same environment. The Psi colloidal solutions of 6 and 9 Pcs were taken to be studied under Pico second $355 \mathrm{~nm}$ laser source. The pumped energy was selected at $3 \mathrm{mj}$ and $6 \mathrm{mj}$ to examine the influence to spontaneous intensity. There is indeed influence in spontaneous signal of all Psi solutions with respect to pump energy. As seen in Figure 11(a), spontaneous light from 6 and 9 Pcs based Psi THF solutions pumped at 3 and $6 \mathrm{mj}$.

The spontaneous signal from the Psi THF, dioxane, and DMSO solutions pumped at $3 \mathrm{mj}$ and $6 \mathrm{mj}$ is exhibited and quite significant. As clearly seen in Figures 11(a)-11(b), signal of Psi THF solution at 6 and 9 Pcs is obtained with variation in intensity that is more pronounced at $6 \mathrm{Pcs}$ when the $3 \mathrm{mj}$ and $6 \mathrm{mj}$ pumped. But the relative intensity is improved almost half with pump energy at $6 \mathrm{mj}$ from $3 \mathrm{mj}$. Psi of 6 and 9 Pcs in dioxane is obtained at large difference in intensity with a bit wide bandwidth. In this case, spontaneous intensity of 9 Pcs solution is observed stronger than 6 Pcs solution as in Figures 11(c)-11(d). The intensity of both solutions is quite improved when the pump energy is at $6 \mathrm{mj}$. The spontaneous emission of 6 and 9 Pcs in DMSO solution is shown in Figures 11(e)11(f). The intensity of both solutions is quite low as compared to the THF and DMSO but observed to be stronger at 9 Pcs. Intensity is also obtained greater at $6 \mathrm{mj}$ pump energy. The influence on spontaneous intensity of all Psi solutions is observed due to the pump energy.

In fact, intensities and band shapes of Psi solutions pumped at $6 \mathrm{mj}$ are quite significant. Among the three Psi solvent solutions, THF and dioxane are stronger in signal than DMSO. The peaks are affected; that may attribute to difference in nature of solvent and distribution of particles in medium. Psi in THF and dioxane are stronger in signal than in DMSO. Present results indicate that high concentration Psi colloidal solution may possibly be synthesized if proper etching employ on silicon surface with larger quantity of Pcs in a specific volume of solvent. Such Si colloidal solutions may help to obtain high emitted light of silicon.

\section{Conclusions}

Porous silicon (Psi) obtained by chemically etching technique is produced under different conditions and successfully separated in organic solvents. The luminescent light of Psi in solvents are examined under UV lamp and found to be depending on the nature of solvents. Psi in polar aprotic and nonpolar solvents observed good luminescent under UV excitation. Most Psi in nonpalar solvent are observed to be unstable with time. Some of them are found highly aggregated and precipitated in polar solvents that do not emit light under UV excitation. Porous silicon is obtained highly crystalline in nature confirmed by XRD. Morphologies and structures of Psi in the solutions are varied solvent to solvent. Most of the Psi particles are uniformly dispersive and have sizes around more or less $5-8 \mathrm{~nm}$ quantifying of silicon wafer piece in etching process which leads to improve PL intensity and also is depending on the etched crosssection area of surface. Thermal effects indicate that PL of nc-Si/Psi decays with respect to temperature and depends on solvents. Spontaneous light of Psi colloidal solutions are quite significant and influenced due to different pump intensity and condition of Psi solutions. The most intense output light is obtained from Psi dioxane solution. Such Psi colloidal solutions will be useful to incorporate into solid active media in order to study light amplification under tunable laser system. This work is still underway.

\section{Conflict of Interests}

The authors declare that there is no conflict of interests regarding the publication of this paper.

\section{Acknowledgment}

This project was supported by NSTIP strategic technologies programs under the Grant no. 10NAN-1037-02 in the Kingdom of Saudi Arabia. The authors extend their appreciation to the Deanship of Scientific Research at King Saud University for funding this work through research group no. RGP-265.

\section{References}

[1] S. Hayashi and K. Yamamoto, "Optical properties of Si-rich $\mathrm{SiO} 2$ films in relation with embedded Si mesoscopic particles," Journal of Luminescence, vol. 70, pp. 352-363, 1996.

[2] B. Kim, D. Kim, C. Lee, and N. Min, "Photoluminescence from nano silicon materials prepared by photoelectrochemical methods," Journal of the Korean Physical Society, vol. 38, no. 3, pp. 245-250, 2001.

[3] T. Canham L, "Silicon quantum wire array fabrication by electrochemical and chemical dissolution of wafers," Applied Physics Letters, vol. 57, article 1046, 1990.

[4] U. Gösele and V. Lehmann, "Light-emitting porous silicon," Materials Chemistry and Physics, vol. 40, no. 4, pp. 253-259, 1995.

[5] W. C. Chan and S. Nie, "Quantum dot bioconjugates for ultrasensitive non-isotopic detection," Science, vol. 281, no. 5385, pp. 2016-2018, 1998. 
[6] M. Bruchez Jr., M. Moronne, P. Gin, S. Weiss, and A. P. Alivisatos, "Semiconductor nanocrystals as fluorescent biological labels," Science, vol. 281, no. 5385, pp. 2013-2016, 1998.

[7] T. D. Lacoste, X. Michalet, F. Pinaud, D. Chemla, A. P. Alivisatos, and S. Weiss, "Ultrahigh resolution multicolor colocalization of single fluorescent probes," Proceedings of the National Academy of Sciences of the United States of America, vol. 97, p. 9461, 2000.

[8] X. Michalet, F. F. Pinaud, L. A. Bentolila et al., "Quantum dots for live cells, in vivo imaging, and diagnostics," Science, vol. 307, no. 5709, pp. 538-544, 2005.

[9] J. K. Jaiswal and S. M. Simon, "Potentials and pitfalls of fluorescent quantum dots for biological imaging," Trends Cell Biology, vol. 14, no. 9, pp. 497-504, 2004.

[10] M. N. Khan and A. S. Al Dwayyan, "Influence of solvent on the physical and lasing properties of dye-doped sol-gel host," Journal of Luminescence, vol. 128, no. 11, pp. 1767-1770, 2008.

[11] S. Yang, W. Cai, G. Liu, H. Zeng, and P. Liu, "Optical study of redox behavior of silicon induced by laser ablation in liquid," Journal of Physical Chemistry C, vol. 113, no. 16, pp. 6480-6484, 2009.

[12] V. Parkhutik, "Porous silicon-mechanisms of growth and applications," Solid-State Electronics, vol. 43, no. 6, pp. 1121-1141, 1999.

[13] D. Dimova-Malinovska, "Application of stain-etched porous silicon in light emitting diodes and cells," Journal of Luminescence, vol. 80, p. 352, 1999.

[14] A. W. Fang, R. Jones, H. Park et al., "Integrated AlGaInAssilicon evanescent racetrack laser and photodetector," Optics Express, vol. 15, no. 5, pp. 2315-2322, 2007.

[15] L. Pavesi, "Routes toward silicon-based lasers," Materials Today, vol. 8, no. 1, pp. 18-25, 2005.

[16] N. Lalic and J. Linnros, "Light emitting diode structure based on Si nanocrystals formed by implantation into thermal oxide," Journal of Luminescence, vol. 80, no. 1-4, pp. 263-267, 1998.

[17] J. R. Walters, G. I. Bourianoff, and H. A. Atwater, "Field-effect electroluminescence in silicon nanocrystals," Nature Materials, vol. 4, article 143, 2005.

[18] L. Zhang, J. L. Coffer, and T. W. Zerda, "Properties of luminescent Si nanoparticles in sol-gel matrices," Journal of Sol-Gel Science and Technology, vol. 11, no. 3, pp. 267-272, 1998.

[19] Y. Posada, L. S. Miguel, L. F. Fonseca et al., "Optical properties of nanocrystalline silicon within silica gel monoliths," Journal of Applied Physics, vol. 96, no. 4, pp. 2240-2243, 2004.

[20] M. N. Khan, A. S. Al Dwayyan, M. S. Al Salhi, and M. Al Hoshan, "Study on characteristics of silicon nanocrystals within sol-gel host," Journal of Experimental Nanoscience, vol. 7, no. 2, pp. 120-132, 2012.

[21] M. N. Khan and A. S. Al Dwayyan, "Influence on structural and PL property of nanocrystals silicon doped sol gel matrix," Journal of Optoelectronics and Advanced Materials, vol. 14, no. 5, p. $448,2012$.

[22] S. A. Al Dwayyan, M. N. Khan, and M. S. Al Salhi, "Optical characterization of chemically etched nanoporous silicon embedded in sol gel matrix," Journal of Nanomaterials, vol. 2012, Article ID 713203, 7 pages, 2012.

[23] M. N. Khan, A. S. Al Dwayyan, and M. S. Al Hoshan, "Morphology and optical properties of a porous silicon-doped sol-gel host," Electronic Materials Letters, vol. 9, no. 5, pp. 697703, 2013.

[24] O. Akcakir, J. Therrien, G. Belomoin et al., "Detection of luminescent single ultrasmall silicon nanoparticles using fluctuation correlation spectroscopy," Applied Physics Letters, vol. 76, no. 14, pp. 1857-1859, 2000.

[25] M. Nayfeh, O. Akcakir, J. Therrien et al., "Highly nonlinear photoluminescence threshold in porous silicon," Applied Physics Letters, vol. 75, no. 26, pp. 4112-4114, 1999.

[26] J. Sarathy, S. Shih, K. Jung et al., "Demonstration of photoluminescence in nonanodized silicon," Applied Physics Letters, vol. 60, no. 13, pp. 1532-1534, 1992.

[27] S. Shih, K. H. Jung, T. Y. Hsieh, J. Sarathy, J. C. Campbell, and D. L. Kwong, "Photoluminescence and formation mechanism of chemically etched silicon," Applied Physics Letters, vol. 60, no. 15, pp. 1863-1865, 1992.

[28] A. Lorusso, V. Nassisi, G. Congedo, N. Lovergine, L. Velardi, and P. Prete, "Pulsed plasma ion source to create Si nanocrystals in $\mathrm{SiO}_{2}$ substrates," Applied Surface Science, vol. 255, no. 10, pp. 5401-5404, 2009.

[29] Table of Prof. Murov's Orgsoltab, which was edited and reposted by Erowid, http://www.organicdivision.org/orig/organic_solvents.html.

[30] J. Tauc, R. Grigorovic, and A. Vancu, "Optical properties and electronic structure of amorphous germanium," Phys Status Solidi B, vol. 15, no. 2, pp. 627-637, 1966.

[31] E. A. Davis and N. F. Mott, "Conduction in non-crystalline systems V. Conductivity, optical absorption and photoconductivity in amorphous semiconductors," Philosophical Magazine, vol. 22, pp. 903-922, 1970.

[32] S. Fellah, R. B. Wehrspohn, N. Gabouze, F. Ozanam, and J. N. Chazalviel, "Photoluminescence quenching of porous silicon in organic solvents: evidence for dielectric effects," Journal of Luminescence, vol. 80, no. 1, pp. 109-113, 1998. 

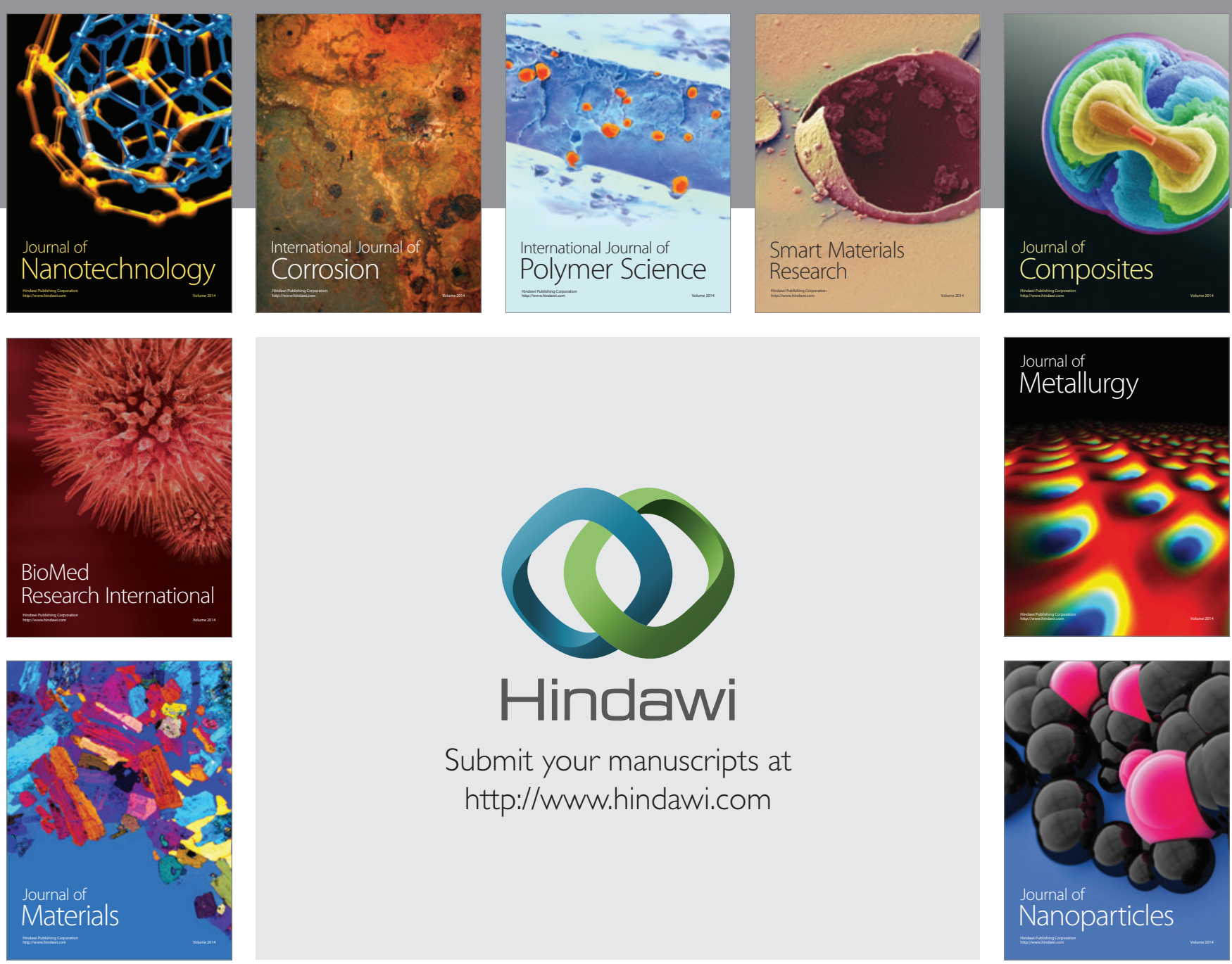

Submit your manuscripts at http://www.hindawi.com
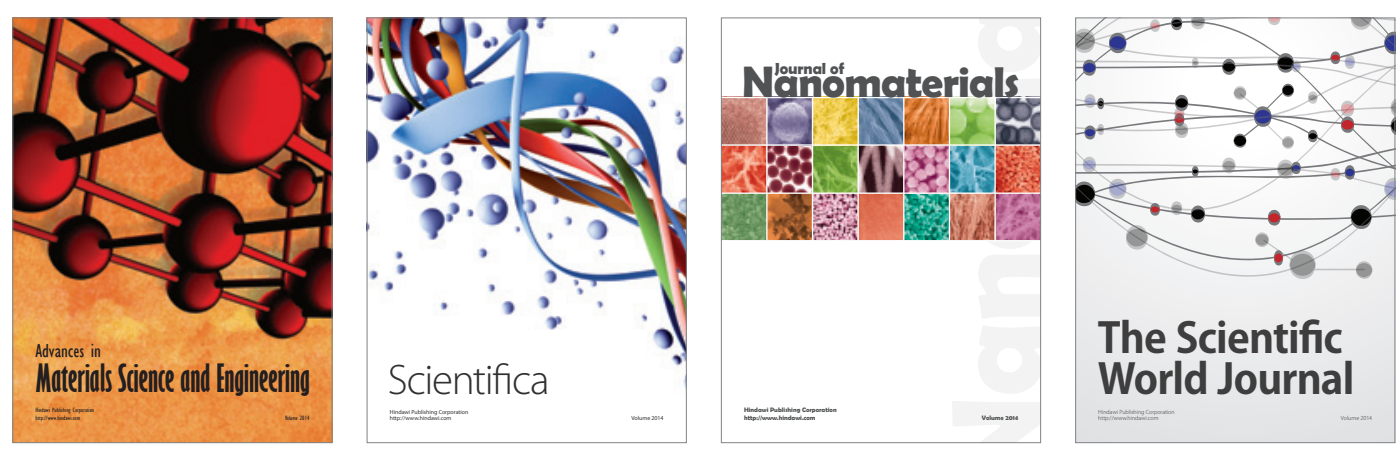

\section{The Scientific World Journal}
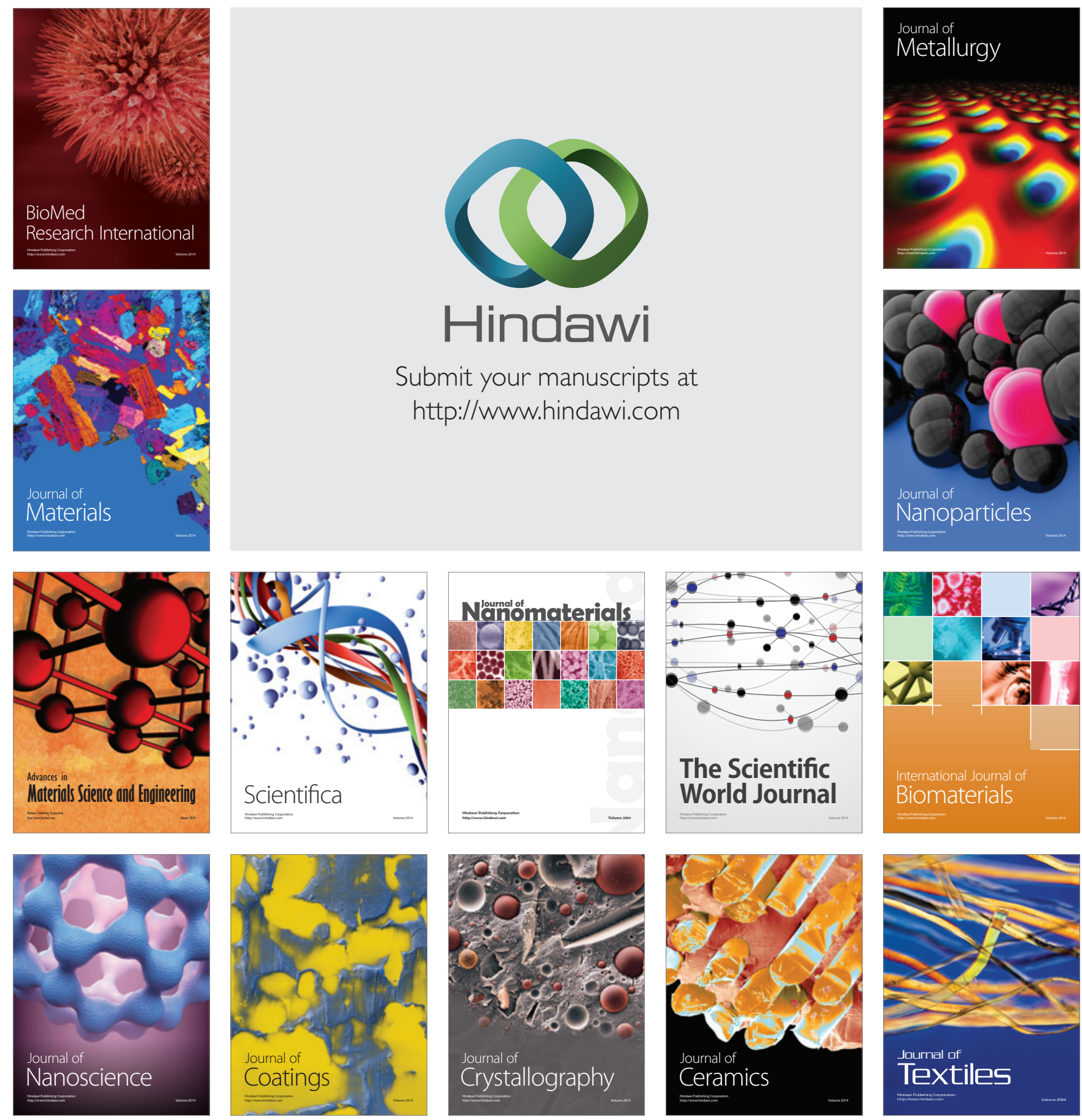